\title{
GENERA OF CURVES ON A VERY GENERAL SURFACE IN $\mathbb{P}^{3}$
}

\author{
C. CILIBERTO, F. FLAMINI, M. ZAIDENBERG
}

\begin{abstract}
In this paper we consider the question of determining the geometric genera of irreducible curves lying on a very general surface $S$ of degree $d \geqslant 5$ in $\mathbb{P}^{3}$ (the cases $d \leqslant 4$ are well known). For all $d \geqslant 4$ we introduce the set $\operatorname{Gaps}(d)$ of all non-negative integers which are not realized as geometric genera of irreducible curves on a very general surface of degree $d$ in $\mathbb{P}^{3}$. We prove that $\operatorname{Gaps}(d)$ is finite and, in particular, that $\operatorname{Gaps}(5)=\{0,1,2\}$. The set $\operatorname{Gaps}(d)$ is the union of finitely many disjoint and separated integer intervals. The first of them, according to a theorem of $\mathrm{Xu}$, is $\operatorname{Gaps}_{0}(d):=\left[0, \frac{d(d-3)}{2}-3\right]$. We show that the next one is $\operatorname{Gaps}_{1}(d):=\left[\frac{d^{2}-3 d+4}{2}, d^{2}-2 d-9\right]$ for all $d \geqslant 6$.
\end{abstract}

\section{Contents}

Introduction

Notation and conventions

1. Preliminaries

2. The range with no gaps

3. Gaps

4. The second gap interval

4.1. A reduction step

4.2. Strategy of the proof

4.3. The cubic case

4.4. The quartic case

References

\section{INTRODUCTION}

In this paper we consider the following question: what are the geometric genera of irreducible curves lying on a sufficiently general surface $S$ of degree $d$ in $\mathbb{P}^{3}$ ?

The answer is trivial for $d \leqslant 3$ : in this case $S$ is rational and carries curves of any genera (see Proposition 1.2 for a more precise result).

For $d \geqslant 4$, the Noether-Lefschetz theorem says that if $S$ is a very general surface of degree $d$ in $\mathbb{P}^{3}$, then all curves on $S$ are complete intersections with another surface in $\mathbb{P}^{3}$ (see $₫ 1$ below). So, in investigating our question, we will suppose $S$ very general in the Noether-Lefschetz sense.

It is well known that on a very general quartic surface in $\mathbb{P}^{3}$ there are curves of all genera (see Corollary 2.2 below). Thus our question starts becoming interesting only for $d \geqslant 5$.

In $\$ 1$ we introduce, for all $d \geqslant 4$, the set $\operatorname{Gaps}(d)$, i.e., the set of all non-negative integers which are not realized as geometric genera of irreducible curves on a very general surface of degree $d$ in $\mathbb{P}^{3}$.

Acknowledgements: The first and second authors have been supported by the Italian MIUR Project protocol 2010S47ARA_005 and by GNSAGA of INdAM. The third author was supported by the French-Italian cooperation project GRIFGA. The authors thank all Institutions which helped them in this collaboration, including their own Departments.

2010 Mathematics Subject Classification: 14N25, 14J70, 32J25, 32Q45.

Key words: projective hypersurfaces, geometric genus, algebraic hyperbolicity. 
By a theorem of $\mathrm{Xu}\left(\right.$ see [27, Thm. 1]), for any $d \geqslant 5$ the integer interval $\operatorname{Gaps}_{0}(d):=\left[0, \frac{d(d-3)}{2}-3\right]$ is contained in $\operatorname{Gaps}(d)$. By contrast, the length 4 interval $J_{0}(d):=\left[\frac{d(d-3)}{2}-2, \frac{d(d-3)}{2}+1\right]$ has empty intersection with Gaps $(d)$ : indeed, it consists of genera of plane sections of $S$, which can have at most 3 nodes if $S$ is general (cf. Proposition 3.3). Similarly, the length 10 interval $J_{1}(d):=\left[d^{2}-2 d-8, d^{2}-2 d+1\right]$ has empty intersection with $\operatorname{Gaps}(d)$ : it consists of genera of quadric sections of $S$, which can have at most 9 nodes if $S$ is general (cf. again Proposition 3.3).

In $\lesseqgtr 2$ we prove that $\operatorname{Gaps}(d)$ is finite and, in particular, that $\operatorname{Gaps}(5)=\operatorname{Gaps}_{0}(5)$ (see Theorem 2.4 and Corollary 2.6). We do not exhibit the minimum $G_{d}$ such that $\operatorname{Gaps}(d) \subseteq\left[0, G_{d}\right]$; so finding $G_{d}$ remains an open problem. However, we provide in Remark 2.5 an asymptotic bound for $G_{d}$.

The proof of the finiteness of Gaps $(d)$ relies on a result by Chiantini and the first author (see [5, Thm. 3.1]) that extends the above discussion on the intervals $J_{0}(d)$ and $J_{1}(d)$. Let $g_{d, n}$ be the arithmetic genus of complete intersections of a surface $S$ of degree $d$ with a surface of degree $n$, and let $\ell_{d, n}$ be the dimension of the linear system of these complete intersections on $S$. Then [5, Thm. 3.1] asserts that for all non-negative integers, the interval $J_{n}(d):=\left[g_{d, n}-\ell_{d, n}, g_{d, n}\right]$ is covered by genera of complete intersections of $S$ with surfaces of degree $n$ with $\delta \in\left[0, \ell_{d, n}\right]$ nodes, lying in reduced components of the Severi variety of nodal curves on $S$. The finiteness of $\operatorname{Gaps}(d)$ follows from the fact that the intervals $J_{n}(d)$ overlap as soon as $n \geqslant d$. In fact this argument proves more, since the curves we find are nodal and lie in reduced components of the Severi variety.

The set Gaps $(d)$ is the union of finitely many disjoint and separated integer intervals, as in (77). Determining all of them is a quite tricky and widely open problem. The proof of the finiteness of Gaps $(d)$ might suggest that these intervals could contain the integer intervals $I_{n}(d):=\left[g_{d, n}+1, g_{d, n+1}-\ell_{d, n+1}-1\right]$, whenever $g_{d, n} \leqslant g_{d, n+1}-\ell_{d, n+1}-2$, which, as we know already, happens only for finitely many $n \geqslant 1$. This is not true in general as shown in [5, Examples 1.1 and 1.2]. However, we prove that this is the case for $d \geqslant 6$ and $n=1$ (see Theorem 4.1). Namely, we show that $I_{1}(d)=\left[\frac{d^{2}-3 d+4}{2}, d^{2}-2 d-9\right]$ is the gap interval $\operatorname{Gaps}_{1}(d)$ next to $\operatorname{Gaps}_{0}(d)$. The proof is not difficult for $d \geqslant 9$ (see Proposition 3.4). It is based on a result by Clemens-Xu-Chiantini-Lopez (see Theorem 3.2), which bounds from below the geometric genus of a complete intersection of a general surface $S$ of degree $d$ in $\mathbb{P}^{3}$ with a surface of degree $n$. When $d \geqslant 9$, this bound forces a curve with geometric genus $g \leqslant d^{2}-2 d-9$ on $S$ to lie on a surface of degree $n \leqslant 2$, and the aforementioned Proposition 3.3 forces $g$ to lie in $J_{0}(d) \cup J_{1}(d)$, which is exactly complementary to $I_{1}(d)$. This argument falls short for $6 \leqslant d \leqslant 8$, which requires a more delicate analysis performed in $\$ 4$, A reduction step in 4.1 reduces these cases to verify non-existence of certain curves on irreducible, but eventually singular surfaces of degree $n=3,4$. This requires in turn a quite subtle case by case analysis which relies on the classification of irreducible cubics and quartics in $\mathbb{P}^{3}$ (see [2, 3, 16, 23, 24, 25]).

The present paper leaves several open problems. The main one, which we mentioned already, would be to have a better comprehension of Gaps $(d)$, of its subdivision (7) into disjoint intervals (how many are there?), and of the constant $G_{d}$ introduced above.

Of course, one might ask similar (and more difficult) questions for general hypersurfaces in higher dimensional projective space, and for complete intersections. Concerning this, it is worthwhile mentioning the result of Chiantini-Lopez-Ran in [7, which implies that the minimal geometric genus of a subvariety on a very general hypersurface of degree $d$ in $\mathbb{P}^{N}$ goes to infinity with $d$, for any given $N \geq 2$.

Notation and conventions. We work over the field of complex numbers. For notation and terminology we refer to [15]. In particular, for $X$ a reduced, irreducible, projective variety, we denote by $\omega_{X}$ its dualizing sheaf: when $X$ is Gorenstein, $\omega_{X}$ is invertible. For divisors on a smooth variety $X$, we use the symbols $\sim$ and $\equiv$ to denote linear and numerical equivalence, respectively. We will sometimes abuse notation and use the same symbol to denote a divisor $D$ on $X$ and its class in $\operatorname{Pic}(X)$. Thus $K_{X}$ will denote a canonical divisor or the canonical sheaf $\omega_{X}$.

Recall that an isolated singular point of a surface $S$ is called a $D u$ Val or irrelevant or simple singularity if its fundamental cycle in a minimal desingularization has dual graph of type $A_{n}, D_{n}, E_{6}, E_{7}, E_{8}$. 


\section{Preliminaries}

Let $d$ be a positive integer. For $\mathcal{L}_{d}:=\left|\mathcal{O}_{\mathbb{P}^{3}}(d)\right|$ we set

$$
N_{d}:=\operatorname{dim}\left(\mathcal{L}_{d}\right)=\left(\begin{array}{c}
d+3 \\
3
\end{array}\right)-1 .
$$

We will denote by $U_{d}$ the dense open subset of $\mathcal{L}_{d}$ whose points correspond to smooth surfaces.

Recall that, by Noether-Lefschetz theorem (see, e.g., [13]), the Picard group of a very general surface $X \in U_{d}$ with $d \geqslant 4$ is generated by $\mathcal{O}_{X}(1)$. Very general means that the property holds off the union $\mathcal{N}_{d}$ of countably many proper Zariski closed subsets of $U_{d}$. The set $\mathcal{N}_{d}$ is called the Noether-Lefschetz locus in degree $d$.

Given $X \in U_{d}$ and a non-negative integer $n$, we let $\mathcal{L}_{X, n}:=\left|\mathcal{O}_{X}(n)\right|$, and we denote by $\ell_{d, n}$ its dimension. One has

$$
\ell_{d, n}=\left\{\begin{array}{cc}
N_{n}=\frac{n\left(n^{2}+6 n+11\right)}{6} & \text { if } n<d \\
N_{n}-N_{n-d}-1=\frac{d\left(3 n^{2}-3 n(d-4)+\left(d^{2}-6 d+11\right)\right)}{6}-1 & \text { if } n \geqslant d,
\end{array}\right.
$$

and

$$
g_{d, n}=\frac{d n(d+n-4)}{2}+1
$$

is the arithmetic genus of the curves in $\mathcal{L}_{X, n}$.

For an irreducible $X \in \mathcal{L}_{d}$ and a non-negative integer $g, \mathcal{V}_{n, g}(X)$ will denote the locally closed subset of $\mathcal{L}_{X, n}$ formed by irreducible curves of geometric genus $g$. If the general member of a component of $\mathcal{V}_{n, g}(X)$ is nodal with $\delta$ nodes, then $g_{d, n}=g+\delta$.

Definition 1.1. Consider the Zariski closure $V_{n, d, g}$ in $U_{d}$ of the locus of all $X \in U_{d}-\mathcal{N}_{d}$ such that $\mathcal{V}_{n, g}(X) \neq \varnothing$. Let $V_{d, g}=\cup_{n \in \mathbb{N}} V_{n, d, g}$. A non-negative integer $g$ is said to be a $d-g a p$ if $V_{d, g} \neq U_{d}$. Roughly speaking, $g$ is a $d$-gap if and only if, for a very general surface $X \in \mathcal{L}_{d}$, one has $\mathcal{V}_{n, g}(X)=\varnothing$ for all $n \geqslant 1$. We will denote by $\operatorname{Gaps}(d)$ the set of $d$-gaps. A non-negative integer $g \notin \operatorname{Gaps}(d)$ will be called a $d-n o n-g a p$.

In studying $\operatorname{Gaps}(d)$ we may and will assume $d \geqslant 4$, since:

Proposition 1.2. $\operatorname{Gaps}(d)=\varnothing$ for $1 \leqslant d \leqslant 3$.

Proof. This is well known in the plane case (see [14, Thm. (1.49)]). For quadrics and cubics the proof is the same. Indeed, $\mathcal{V}_{n, 0}(X)$ is nonempty for $X \in U_{d}$, with $d \leqslant 3$ (see, e.g., [22]). Then a well known deformation argument shows that $\mathcal{V}_{n, g}(X)$ is nonempty for all $g \leqslant g_{d, n}$ (see, e.g., [18]).

We will abuse notation and, for integers $k, l$ with $k \leqslant l$, we will write $[k, l]$ for the integer interval $[k, l] \cap \mathbb{Z}$, and we call it simply interval.

By [27, Thm. 1], every $g \leqslant g_{d, 1}-\ell_{d, 1}-1=g_{d, 1}-4=\frac{d(d-3)}{2}-3$ is a $d$-gap, i.e.

$$
\operatorname{Gaps}_{0}(d):=\left[0, \frac{d(d-3)}{2}-3\right] \subseteq \operatorname{Gaps}(d) .
$$

$\operatorname{Gaps}_{0}(d)$ will be called the initial gap interval (see Remark 2.3 below).

\section{The RANGE WITH NO GAPS}

In this section we will show the finiteness of $\operatorname{Gaps}(d)$ for any $d \geqslant 4$. To do this, we first recall:

Theorem 2.1. ([5, Thm. 3.1]) Let $X \in \mathcal{L}_{d}$ be a general surface of degree $d \geqslant 4$ in $\mathbb{P}^{3}$. For all integers $n \geqslant 1$ and $g \in J_{n}(d):=\left[g_{d, n}-\ell_{d, n}, g_{d, n}\right]$ there is a reduced, irreducible component $\mathcal{V}$ of $\mathcal{V}_{n, g}(X)$ whose general element is nodal with $\delta=g_{d, n}-g$ nodes and $\operatorname{dim}(\mathcal{V})=\ell_{d, n}-\delta$. In particular $J_{n}(d) \cap \operatorname{Gaps}(d)=\varnothing$.

Theorem 2.1 is stated in [5] under the assumption $n \geqslant d$, but the same (actually easier) argument works for $n<d$.

The following consequence is well known: 
Corollary 2.2. One has $\operatorname{Gaps}(4)=\varnothing$.

Proof. This follows from Theorem 2.1, since $g_{4, n}=\ell_{4, n}$ for all positive integers $n$.

Remark 2.3. Since $\ell_{d, 1}=3$, from Theorem 2.1 one has $\mathcal{V}_{1, g}(X) \neq \varnothing$ for $g \in J_{1}(d)=\left[g_{d, 1}-3, g_{d, 1}\right]$, i.e., any such $g$ is a $d$-non-gap.

As a consequence of Theorem 2.1, we obtain:

Theorem 2.4. For integers $n \geqslant d \geqslant 4$, and for any $g \geqslant g_{d, n-1}-\ell_{d, n-1}$, there is an irreducible nodal curve of geometric genus $g$ on the general surface of degree $d$ in $\mathbb{P}^{3}$. In particular, one has Gaps $(d) \subset$ $\left[0, g_{d, d-1}-\ell_{d, d-1}-1\right]=\left[0, \frac{d(d-1)(5 d-19)}{6}-1\right]$.

Proof. By Theorem 2.1, we need to show that for $n \geqslant d$, the union $J_{n-1}(d) \cup J_{n}(d)$ is an integer interval, i.e.,

By (21), (15) reads

$$
\ell_{d, n} \geqslant g_{d, n}-g_{d, n-1}-1=\frac{d(2 n+d-5)}{2}-1 .
$$

which holds for $n \geqslant d$.

$$
3 n(n-d+2)+\left(d^{2}-9 d+26\right) \geqslant 0,
$$

Remark 2.5. It is possible to give a better estimate for the minimal integer $G_{d}$ such that $\operatorname{Gaps}(d) \subset\left[0, G_{d}\right]$. Indeed, if $n<d$, then (5) reads

$$
n^{3}+6 n^{2}+n(11-6 d)-3\left(d^{2}-5 d-2\right) \geqslant 0 .
$$

For this to hold, it suffices that

$$
n^{3}+6 n^{2}-6 n d-3 d^{2} \geqslant 0 \text { i.e. } d \leqslant \sqrt{\frac{n^{3}+9 n^{2}}{3}}-n .
$$

The latter inequality is fulfilled, e.g., if $d>n \geqslant \sqrt[3]{12 d^{2}}$. For any such $n$, we have $G_{d} \leq g_{d, n-1}-\ell_{d, n-1}-1$.

Corollary 2.6. For any integer $g \geqslant 3$ there is an irreducible nodal curve of geometric genus $g$ on a general surface of degree 5 in $\mathbb{P}^{3}$, i.e., $\operatorname{Gaps}(5)=\operatorname{Gaps}_{0}(5)=\{0,1,2\}$.

Proof. We know that (5) holds for any $n \geqslant 5=d$. When $2 \leqslant n \leqslant 4$, (6) also holds. Thus $G_{5} \leq g_{5,1}-\ell_{5,1}-1=$ 2 (see Remarks 2.3 and 2.5), and so $\operatorname{Gaps}(5) \subseteq\{0,1,2\}$. On the other hand, $\operatorname{Gaps}_{0}(5)=\{0,1,2\}$ by Xu's theorem cited above.

\section{GAPS}

By Proposition 1.2 and Corollaries 2.2 and 2.6, we can focus on $d \geqslant 6$. By Theorem 2.4, Gaps $(d)$ is finite. Hence there exists an integer $n_{d} \geqslant 0$ such that

$$
\operatorname{Gaps}(d)=\bigcup_{j=0}^{n_{d}} \operatorname{Gaps}_{j}(d), \text { with } \operatorname{Gaps}_{j}(d):=\left[a_{j}, b_{j}\right],
$$

where

$$
a_{0}=0<b_{0}=\frac{d(d-3)}{2}-3 \text { and } b_{j-1}+1<a_{j} \leqslant b_{j} \text { for all } j>0 .
$$

The disjoint and separated intervals $\operatorname{Gaps}_{j}(d)$ are called the gap intervals. The initial gap interval $\operatorname{Gaps}_{0}(d)$ is as in (4). Our aim is to determine the next gap interval $\operatorname{Gaps}_{1}(d)$, see Theorem 4.1 below.

Remark 3.1. By Theorem 2.4 we have

$$
\operatorname{Gaps}(d) \subset \mathbb{N} \cup\{0\} \backslash \bigcup_{n \geq 1} J_{n}(d) .
$$

Looking at the proof of this theorem, one might guess that there are $d$-gaps $g$ with

$$
g_{d, n-1}+1 \leqslant g \leqslant g_{d, n}-\ell_{d, n}-1
$$


for any $n \geqslant 2$, any time (5) does not hold, namely that

$$
I_{n-1}(d):=\left[g_{d, n-1}+1, g_{d, n}-\ell_{d, n}-1\right] \subseteq \operatorname{Gaps}(d)
$$

if $n \geqslant 2, d \geqslant 6$ and (5) does not hold. However, this is not true in general, as [5, Examples 1.1 and 1.2] show. For instance, $I_{2}(20) \nsubseteq \operatorname{Gaps}(20)$, by [5, Example 1.1]. Nonetheless, $I_{1}(d) \subseteq \operatorname{Gaps}(d)$, see Theorem 4.1 below.

Determining all $d$-gaps for $d \geqslant 6$ is a tricky problem. In this section we show that there are $d$-gaps other than the ones in $\operatorname{Gaps}_{0}(d)$, i.e. $n_{d}>0$ for $d \geqslant 6$. Recall first the following results.

Theorem 3.2. ([6, Thm. (1.2)], [10, [27, Thm. 2.1]) Let $X$ be a general surface of degree $d \geqslant 5$ in $\mathbb{P}^{3}$, and let $C \in \mathcal{L}_{X, n}$ be an irreducible curve of geometric genus $g$. Then

$$
g>\frac{n d(d-5)}{2}+1
$$

Proposition 3.3. ([9, Cor. 2.9]) Let $X$ be a general surface of degree $d \geqslant 3$ in $\mathbb{P}^{3}$. If $g \geqslant 0$ and $n \in\{1,2\}$ are such that $\mathcal{V}_{n, g}$ is non-empty, then

$$
g_{d, 1}-3 \leqslant g \leqslant g_{d, 1} \quad \text { if } \quad n=1 \quad \text { and } \quad g_{d, 2}-9 \leqslant g \leqslant g_{d, 2} \quad \text { if } \quad n=2 .
$$

As a consequence, recalling (10), we have:

Proposition 3.4. If $d \geqslant 9$ then

$$
\operatorname{Gaps}_{1}(d)=I_{1}(d)=\left[\frac{d^{2}-3 d+4}{2}, d^{2}-2 d-9\right] .
$$

Furthermore, for $d=7,8$ we have

$$
\left[g_{7,1}+1, g_{7,1}+7\right] \subseteq \operatorname{Gaps}_{1}(7) \text { and }\left[g_{8,1}+1, g_{8,1}+16\right] \subseteq \operatorname{Gaps}_{1}(8) .
$$

Proof. Let $X$ be a very general surface of degree $d \geqslant 9$ in $\mathbb{P}^{3}$. Let $C \in \mathcal{L}_{X, n}$ be an irreducible curve with geometric genus $g \leqslant g_{d, 2}-\ell_{d, 2}-1=d(d-2)-9$. By Theorem 3.2. we have

$$
d(d-2)-9 \geqslant g>\frac{n d(d-5)}{2}+1, \text { i.e., } n<\frac{2\left(d^{2}-2 d-10\right)}{d(d-5)} .
$$

Suppose that $n \geqslant 3$. Then (14) yields $d^{2}-11 d+20<0$, which implies $d \leqslant 8$, a contradiction. Thus $n \leqslant 2$ and by Proposition 3.3 one has $I_{1}(d) \subseteq \operatorname{Gaps}_{1}(d)$. Since $\ell_{d, 2}=9$, by Theorem 2.1 we have $\mathcal{V}_{2, g_{d, 2}-9}(X) \neq \varnothing$, i.e., $g_{d, 2}-9$ is a $d$-non-gap. Since $g_{d, 1}$ is also a $d-$ non-gap, the equality in (12) follows.

For $d=7,8$ the argument is similar; we leave the details to the reader.

\section{The SECOND GAP INTERVAL}

In this section we extend Proposition 3.4, proving the following theorem.

Theorem 4.1. For all $d \geq 6$ one has

$$
\operatorname{Gaps}_{1}(d)=I_{1}(d)=\left[\frac{d^{2}-3 d+4}{2}, d^{2}-2 d-9\right] .
$$

By Proposition 3.4, we may assume in the sequel that $6 \leqslant d \leqslant 8$. The proof consists in a case by case analysis, which we will perform in the rest of this section. 
4.1. A reduction step. The following lemma reduces the analysis to finitely many cases:

Lemma 4.2. Assume $6 \leqslant d \leqslant 8$. To prove that $I_{1}(d)=\operatorname{Gaps}_{1}(d)$, it suffices to show that for $X \in \mathcal{L}_{d}$ general, one has $\mathcal{V}_{n, g}(X)=\varnothing$ if

$$
\begin{array}{lll}
d=6, & n=3, & g \in[11,15] \\
d=6, & n=4, & g \in[14,15] \\
d=7, & n=3, & g \in[23,26] \\
d=8, & n=3, & g \in[38,39] .
\end{array}
$$

Proof. For $d=6,7,8$, from (8) we derive the inclusions $\operatorname{Gaps}_{1}(d) \subset I_{1}(d)$, where $I_{1}(6)=[11,15], I_{1}(7)=$ $[16,26]$, and $I_{1}(8)=[22,39]$, see (10). To show the inverse inclusions, we have to check that every $g \in I_{1}(d)$ is a $d$-gap for $d=6,7,8$.

Suppose $\mathcal{V}_{n, g}(X) \neq \varnothing$ for $X \in \mathcal{L}_{d}$ general. Since the intervals in (11) do not meet $I_{1}(d)$, from Proposition 3.3 and $d \geqslant 6$ it suffices to restrict to $n \geqslant 3$. On the other hand, if $n \geqslant 5$, (14) gives $5 \leqslant n<\frac{2\left(d^{2}-2 d-10\right)}{d(d-5)}$, i.e., $3 d^{2}-21 d+20<0$, which contradicts $d \geqslant 6$. Thus it is enough to consider $n \in\{3,4\}$. The remaining possibilities are as follows.

- $(d, n)=(6,3)$ and $g \in I_{1}(6)$ as in the first line of (15);

- $(d, n)=(7,3)$; then by (13), $[16,22] \subseteq \operatorname{Gaps}_{1}(7)$. Hence it remains to eliminate the values of $g$ as in the third line of (15);

- $(d, n)=(8,3)$; using again (12) and (13), one reduces to the last line of (15);

- if $n=4$, (14) yields $2 d^{2}-16 d+20<0$. So the only possibility is $d=6$. From Theorem 3.2 we deduce $g>13$, which leaves the range of $g$ as in the second line of (15).

4.2. Strategy of the proof. By Lemma 4.2 we need to show that $\mathcal{V}_{n, g}(X)=\varnothing$ for $X \in \mathcal{L}_{d}$ general and $d$, $n$ and $g$ as in (15). A basic ingredient will be the following result.

Proposition 4.3. (see [9, Prop. 2.8]) Let $S$ be a smooth projective surface, $\mathcal{H}$ the Hilbert scheme of curves on $S$, and $\mathcal{V}_{g}$ the locally closed subset of $\mathcal{H}$ formed by irreducible curves of geometric genus $g$. For any component $\mathcal{V} \subseteq \mathcal{V}_{g}$ we set

$$
v:=\operatorname{dim}(\mathcal{V}) \text { and } \kappa:=\mathrm{K}_{\mathrm{S}} \cdot \Gamma
$$

where $\Gamma$ corresponds to a general point in $\mathcal{V}$. Then $v \leqslant v_{0}:=\max \{g, g-1-\kappa\}$.

Let $d, n$ and $g$ be as in (15). Let $\mathcal{F} \subseteq \mathcal{L}_{n}$ be an irreducible closed subset, which is the parameter space for a flat family of surfaces in $\mathbb{P}^{3}$ of degree $n$. We assume that its general point corresponds to an irreducible surface $\Sigma$.

Consider the incidence relation $I \subseteq U_{d} \times \mathcal{F}$, consisting of all pairs $(X, \Sigma)$ such that $X$ and $\Sigma$ intersect along a reduced, irreducible curve $\Gamma$ of geometric genus $g$. Then $I$ is locally closed with the projections

$$
p: I \rightarrow \mathcal{U}_{d} \text { and } q: I \rightarrow \mathcal{F} .
$$

If $I^{\prime}$ is an irreducible component of $I$ which dominates $\mathcal{F}$ via $q$, then

$$
\operatorname{dim}\left(I^{\prime}\right)=\operatorname{dim}(\mathcal{F})+\operatorname{dim}\left(q^{-1}(\Sigma) \cap I^{\prime}\right) .
$$

The main point in our strategy is to estimate $\operatorname{dim}\left(q^{-1}(\Sigma) \cap I^{\prime}\right)$. If $(X, \Sigma) \in I^{\prime}$ and $C$ is the intersection of $X$ and $\Sigma$, one has a linear system of dimension $N_{d-n}+1$ of surfaces of degree $d$ containing $C$. Thus we get a $\left(N_{d-n}+1\right)$-dimensional set of pairs $(X, \Sigma) \in I^{\prime}$ such that the intersection of $X$ and $\Sigma$ is $C$.

Let $\mathcal{V}_{d, g}(\Sigma)$ be the locally closed subset of $\mathcal{L}_{\Sigma, d}:=\left|\mathcal{O}_{\Sigma}(d)\right|$ formed by irreducible curves of geometric genus $g$, and let $\mathcal{V} \subseteq \mathcal{V}_{d, g}(\Sigma)$ be any of its irreducible component. Applying Proposition 4.3 to the minimal desingularization

$$
\pi: S \rightarrow \Sigma \subset \mathbb{P}^{3}
$$

of $\Sigma$, we obtain the bound

$$
\operatorname{dim}(\mathcal{V}) \leqslant v_{0} .
$$


Therefore, for $\Sigma \in \mathcal{F}$ general,

Hence by (16),

$$
\operatorname{dim}\left(q^{-1}(\Sigma) \cap I^{\prime}\right) \leqslant v_{0}+N_{d-n}+1
$$

$$
\operatorname{dim}\left(I^{\prime}\right) \leqslant \operatorname{dim}(\mathcal{F})+v_{0}+N_{d-n}+1 .
$$

To prove that $\mathcal{V}_{n, g}(X)=\varnothing$ for $X \in U_{d}$ general, one needs to prove that $\left.p\right|_{I^{\prime}}$ is not dominant onto $U_{d}$, i.e.,

$$
\operatorname{dim}\left(I^{\prime}\right)<N_{d}
$$

for any $I^{\prime}$ as above. Thus (20) yields the following sufficient condition for (21) to hold:

$$
\operatorname{dim}(\mathcal{F})+v_{0}<\Phi(n, d):=N_{d}-N_{d-n}-1=\left\{\begin{array}{cc}
\frac{3}{2} d(d+1) & \text { if } n=3 \\
2 d^{2}+1 & \text { if } n=4
\end{array}\right.
$$

for all pairs $(d, n)$ as in (15).

The proof of Theorem 4.1 reduces to check (22) for all possible parameter spaces $\mathcal{F}$ of cubics (resp., of quartics) in $\mathbb{P}^{3}$, whose general element $\Sigma$ is irreducible.

4.3. The cubic case. Classification of irreducible cubic surfaces in $\mathbb{P}^{3}$ started a century and a half ago by Schläfli in [20] and Cayley in [4], see e.g., [12] for a historical account and references. About one hundred years later, Bruce and Wall (see [2, 3]) reconsidered this classification via the modern theory of singularities.

Description 4.4. Let $\Sigma \subset \mathbb{P}^{3}$ be an irreducible cubic surface. Then:

(i) either $\Sigma$ has at most Du Val singularities, or

(ii) it is a cone over a plane cubic, or

(iii) it is a scroll which is not a cone.

Case (i) occurs for a general $\Sigma \in \mathcal{L}_{3}$; recall that $N_{3}=\operatorname{dim}\left(\mathcal{L}_{3}\right)=19$, see (11).

In case (ii) we will denote by $\mathcal{C}$ the irreducible closed subvariety of $\mathcal{L}_{3}$, whose general point corresponds to a cone over a smooth, plane cubic. Clearly $\operatorname{dim}(\mathcal{C})=12$.

In case (iii) we will denote by $\mathcal{R}$ the irreducible closed subvariety of $\mathcal{L}_{3}$, whose general point corresponds to an irreducible scroll $\Sigma$ which is not a cone. Such a scroll $\Sigma$ appears as the general projection in $\mathbb{P}^{3}$ of a smooth rational normal scroll $S$ of degree 3 in $\mathbb{P}^{4}$, and $\operatorname{Sing}(\Sigma)$ is a double line (see e.g., [3]). An easy parameter count, which can be left to the reader, shows that $\operatorname{dim}(\mathcal{R})=13$.

We keep the notation of $\S 4.2$. To prove that $\mathcal{V}_{3, g}(X)=\varnothing$ for $X \in \mathcal{L}_{d}$ general and $d$ and $g$ for all cases with $n=3$, i.e., as in the first, third and fourth line of (15), we will show that (22) holds. One has to analyse cases (i) - (iii) of Description 4.4 occurring for $\Sigma$ corresponding to the general point of $\mathcal{F}$.

We will denote by $H$ the hyperplane section class of $\Sigma$. By abuse of notation we will also denote by $H$ its total transform on the minimal desingularization $S$ of $\Sigma$ as in (17).

4.3.1. Case (i). We have $\omega_{\Sigma} \cong \mathcal{O}_{\Sigma}(-H)$ and $K_{S}=\pi^{*}\left(K_{\Sigma}\right)=-H$ (see, e.g., [1, Lemma 1.2.2]).

Let $\mathcal{V} \subseteq \mathcal{V}_{d, g}(\Sigma)$ be an irreducible component, let $C$ be the curve corresponding to its general point, and let $\Gamma$ be the proper transform of $C$ on $S$. Then $\Gamma \sim d H-D$, where $D \geqslant 0$ is a $\pi$-exceptional divisor, which takes into account if $C$ passes through some of the singularities of $\Sigma$. Since every irreducible component of $D$ is a $(-2)$-curve, $D \cdot K_{S}=0$. Hence $\kappa=K_{S} \cdot \Gamma=-3 d$.

By (18) and Proposition 4.3 we have $\operatorname{dim}(\mathcal{V}) \leqslant v_{0}=3 d+g-1$. Since $\operatorname{dim}(\mathcal{F}) \leqslant 19$, a sufficient condition for (22) to hold is

$$
3 d^{2}-3 d-36>2 g \text {. }
$$

Then (23) holds for all the $(d, g)$ in (15) which correspond to $n=3$. Hence the same is true for (22).

4.3.2. Case (ii). One has

$$
\operatorname{dim}(\mathcal{F}) \leqslant \operatorname{dim}(\mathcal{C})=12 .
$$

Let $Y \subset \mathbb{P}^{2}$ be the plane cubic which is the base of the cone $\Sigma$. There are the following possibilities: (a) $Y$ is smooth; (b) $Y$ is nodal; (c) $Y$ is cuspidal. We will discuss cases (a) and (b) only, since (c) is similar to (b) and can be left to the reader. 
4.3.3. Case (ii,a). The minimal desingularization of $\Sigma$ is $S=\mathbb{P}_{Y}\left(\mathcal{O}_{Y} \oplus \mathcal{O}_{Y}(1)\right)$, which is a ruled surface with base $Y$. We denote by $F$ the numerical equivalence class of a fibre of the structure morphism $\sigma: S \rightarrow Y$ and by $E$ the section with $E^{2}=-3$ which is contracted to the vertex $\mathfrak{v}$ of $\Sigma$. One has $H \equiv E+3 F$ and $K_{S} \equiv-E-H$. With the usual notation, let $\Gamma$ be the proper transform of $C \in \mathcal{V}_{d, g}(\Sigma)$ on $S$.

(†) If $C$ does not pass through $\mathfrak{v}$, then $\Gamma \equiv d H, \kappa=K_{S} \cdot \Gamma=-3 d$, and by Proposition 4.3 we find the upper bound $v_{0}=3 d+g-1$ in (18). Then the discussion proceeds as in case (i) above, with the same conclusion. ( $\ddagger$ ) If $C$ passes through $\mathfrak{v}$, then one has $\Gamma \equiv d H-E$. Indeed, a priori one has $\Gamma \equiv d H-m E$. On the other hand, since $X$ is smooth at $\mathfrak{v}$, the general ruling of the cone intersects $X$ at $d-1$ points off $\mathfrak{v}$; hence $\Gamma \cdot F=d-1$, which proves $m=1$ (the same holds in all cases below, dealing with cones).

Thus $\kappa=K_{S} \cdot \Gamma=-3 d-3$. By Proposition 4.3, we find $v_{0}=g+3 d+2$. Taking into account (24) and proceeding as in case (i), one sees that (22) holds in all cases of (15) with $n=3$.

4.3.4. Case (ii,b). As before, (24) holds. The minimal desingularization $S$ of $\Sigma$ is the Hirzebruch surface $\mathbb{F}_{3}=\mathbb{P}\left(\mathcal{O}_{\mathbb{P}^{1}} \oplus \mathcal{O}_{\mathbb{P}^{1}}(-3)\right)$. We denote again by $F$ the numerical equivalence class of a fibre of the structure morphism $\sigma: S \rightarrow \mathbb{P}^{1}$ and by $E$ the section with $E^{2}=-3$ which is contracted to the vertex $\mathfrak{v}$ of $\Sigma$. One has $H \equiv E+3 F$ and $K_{S} \equiv-2 H+F$.

Let $\Gamma$ and $C$ be as usual. Two cases have to be analyzed.

(†) If $C$ does not pass through $\mathfrak{v}$, then $\Gamma \equiv d H$. One has $\kappa=K_{S} \cdot \Gamma=-5 d$ and $v_{0}=5 d+g-1$. By (24), to prove that (22) holds, it suffices to show that $3 d^{2}-7 d-22>2 g$ for all $d$ and $g$ in (15) with $n=3$. A direct computation confirms that this is the case.

$(\ddagger) C$ passes through $\mathfrak{v}$. As in case (ii,a) $-(\ddagger)$, one has $\Gamma \equiv d H-E$. Thus, $\kappa=K_{S} \cdot \Gamma=-5 d-1$ and similar computations as in the previous case show that (22) holds.

4.3.5. Case (iii). One has

$$
\operatorname{dim}(\mathcal{F}) \leqslant \operatorname{dim}(\mathcal{R})=13
$$

The minimal desingularization $S$ of $\Sigma$ is isomorphic to $\mathbb{F}_{1}$, with the structure map $\sigma: S \rightarrow \mathbb{P}^{1}$. We denote by $E$ the section of $S$ with $E^{2}=-1$ and with $F$ a fibre. Then $H \equiv E+2 F$ and $K_{S} \equiv-2 E-3 F$. Since $\Gamma \equiv d H$, we get $\kappa=K_{S} \cdot \Gamma=-5 d$, hence we find $v_{0}=5 d+g-1$ as in case (ii,b)-(†). By (25), to prove that (22) holds it suffices to verify that $3 d^{2}-7 d-24>2 g$, for all $d$ and $g$ in (15) with $n=3$. A direct check shows that this is the case.

In conclusion, the above analysis shows that, for $X \in U_{d}$ general, one has $\mathcal{V}_{n, g}(X)=\varnothing$ for $d, n, g$ as in (15) with $n=3$.

4.4. The quartic case. The only case left from (15) is $d=6, n=4, g \in[14,15]$. To finish the proof of Theorem 4.1 we have to verify that for $X \in U_{6}$ general, one has $\mathcal{V}_{4, g}(X)=\varnothing$ for $g \in\{14,15\}$.

We will keep the notation as in $\$ 4.2$. From (22), one has $\Phi(4,6)=73$ and $\operatorname{dim}(\mathcal{F}) \leqslant 34=\operatorname{dim}\left(\mathcal{L}_{4}\right)$. Therefore, for (22) to hold, it suffices to prove the upper bound

$$
v_{0} \leqslant 39 \text {. }
$$

This is what we will do for all cases discussed below, except the last one, where the argument is different.

4.4.1. Classification of quartic surfaces. The classification of irreducible quartic surfaces in $\mathbb{P}^{3}$ is as old as that of cubics, see e.g., [12]. Similarly as for cubics, we will use a modern version elaborated in [16, 23, 24, 25], which we shortly recall here. For any such quartic $\Sigma$ one has $\omega_{\Sigma} \cong \mathcal{O}_{\Sigma}$. As usual, we let $\pi: S \rightarrow \Sigma$ be the minimal desingularization, and we keep the notation as in the cubic case.

First we treat the case $\Sigma$ normal (see [23, 24]). If $p \in \operatorname{Sing}(\Sigma)$, the geometric genus of $p$ is defined to be

$$
p_{g}(p)=\operatorname{dim}_{\mathbb{C}}\left(\left(R^{1} \pi_{*} \mathcal{O}_{S}\right)_{p}\right)
$$

(see [26] or [23, Def. 1])). We set

$$
\operatorname{Irrat}(\Sigma):=\left\{p \in \operatorname{Sing}(\Sigma) \mid p_{g}(p)>0\right\},
$$

which is the set of irrational singularities of $\Sigma$. 
Proposition 4.5. (cf. [23, Propositions 5,7,8, Theorem 1]) Let $\Sigma \subset \mathbb{P}^{3}$ be a normal, irreducible quartic surface. One has:

(a) if $p \in \operatorname{Sing}(\Sigma)$ and $p_{g}(p)=0$, then $p$ is a Du Val singularity;

(b) there exists a unique effective divisor $E$ on $S$ such that $\mathcal{O}_{S}(E) \cong \omega_{S}^{\vee}$.

Moreover:

(i) if $\operatorname{Irrat}(\Sigma)=\varnothing$, then $S$ is a K3 surface, i.e., $E=0$;

(ii) if $\operatorname{Irrat}(\Sigma) \neq \varnothing$, then $S$ is birationally equivalent to a ruled surface, $E>0$ and its connected components bijectively correspond via $\pi$ to singularities in $\operatorname{Irrat}(\Sigma)$.

Furthermore, if $q:=h^{1}\left(\mathcal{O}_{S}\right)$, then:

(ii-1) if $q \neq 1$, then $\operatorname{Irrat}(\Sigma)$ consists of a single point $p$ such that $p_{g}(p)=q+1$;

(ii-2) if $q=1$, then $\operatorname{Irrat}(\Sigma)$ consists either of one point $p$, with $p_{g}(p)=2$, or of two points $p_{i}$, for $i=1,2$, with $p_{g}\left(p_{i}\right)=1$, that are both simple elliptic, i.e., $\pi^{-1}\left(p_{i}\right)$ is a smooth, irreducible elliptic curve.

In case (ii) of Proposition 4.5, i.e., when $\Sigma$ is a normal quartic surface in $\mathbb{P}^{3}$ with irrational singular points, there is a detailed classification in [16, which we will need to go through later. It can be briefly summarized as follows.

Proposition 4.6. Let $\Sigma \subset \mathbb{P}^{3}$ be a normal, irreducible quartic surface such that $\operatorname{Irrat}(\Sigma) \neq \varnothing$. Then either (i) $q=0$ and $\Sigma$ is rational, or

(ii) $q=1$ and $\Sigma$ is birational to an elliptic ruled surface or

(iii) $q=3$ and $\Sigma$ is a cone over a smooth plane quartic curve.

As for the non-normal case, we have:

Proposition 4.7. (cf. [25, Lemma 2.3]) Let $\Sigma \subset \mathbb{P}^{3}$ be a non-normal, irreducible quartic surface. Then $S$ is either a scroll over a smooth curve of genus 2 , or an elliptic scroll or a rational surface.

We will examine the various cases, first treating the normal, then the non-normal ones. Remember that to accomplish the proof of Theorem 4.1] it suffices to establish inequality (26).

4.4.2. The K3 case. This is case (i) in Proposition 4.5, Then $v_{0}=g \leqslant 15$ (cf. Proposition 4.3). So (26) holds.

4.4.3. Normal quartic surfaces with an irrational singularity. Next we turn to case (ii) in Proposition 4.5. which, according to Proposition [4.6, gives rise to various subcases. We refer to [16] for details. To make the reading more accessible, let us first overview the terminology and the main classification principle in [16]. The latter uses the triplets $(X, B, G)$ consisting in a smooth, projective surface $X$, a smooth nonhyperelliptic curve $B$ of genus 3 on $X$, and an effective anticanonical divisor $G \in\left|-K_{X}\right|$, where $G \neq 0$. Such a triplet satisfies condition $\mathcal{C}_{r}$ if $K_{X}+B$ is nef and $B \cdot G=r$. If $r \geq 1$, then blowing up $\sigma: X^{\prime} \rightarrow X$ at a point of $B \cap G$ leads to a $\mathcal{C}_{r-1}$-triplet $\left(X^{\prime}, B^{\prime}, G^{\prime}\right)$ with $B^{\prime}=\sigma^{*}(B)-F$ and $G^{\prime}=\sigma^{*}(B)-F$, where $F$ is the exceptional (-1)-curve. After $r$ blowups one has a birational morphism $\rho: S \rightarrow X$ and one arrives at a $\mathcal{C}_{0}$-triplet $(S, H, E)$ where

$$
H:=\rho^{*}(B)-\Delta \text { and } E:=\rho^{*}(G)-\Delta,
$$

where $\Delta$ is the total $\rho$-exceptional divisor. This process is called separation ([16, p. 947]) and $(S, H, E)$ is called the (result of the) separation of $(X, B, G)$. Notice that the divisor $E$ can be reducible and/or non-reduced, even if $G$ is reduced and irreducible. This may happen if $G$ is singular and $r \geq 1$.

One says that a $\mathcal{C}_{0}$-triplet $(S, H, E)$ is a basic triplet (our terminology here slightly differs from the one in [16]), if $H$ meets every (-1)-curve on $S$. There exists a classification of all basic triplets into 4 types $A, B, C, D$ ([16, Theorem 1.7]), together with a list of examples of each type ([16, §2]) obtained via separation, that we will permanently address below. The main theorem in [16, §3] asserts that this list is exhaustive, and so describes all the normal quartic surfaces in $\mathbb{P}^{3}$ with irrational singularities. Together with [16, Prop. 1.4], this yields the following theorem. 
Theorem 4.8. Any basic triplet $(S, H, E)$ arises as the minimal desingularization $\pi=\varphi_{|H|}: S \rightarrow \Sigma$ of a normal quartic surface $\Sigma$ in $\mathbb{P}^{3}$ with irrational singular points, where $H$ is the pullback of a hyperplane section of $\Sigma$ which does not pass through any irrational singular point, and $E$ is an effective $\pi$-exceptional anticanonical divisor on $S$. Conversely, the minimal desingularization $\pi: S \rightarrow \Sigma$ of a normal quartic surface $\Sigma$ in $\mathbb{P}^{3}$ with an irrational singular point yields a basic triplet $(S, H, E)$, with $H$ and $E$ as before.

Keeping notation as in $\S 4.2$, let $C$ be an irreducible curve on $\Sigma$ cut out by a smooth sextic surface $X$ in $\mathbb{P}^{3}$, and let $\Gamma$ be its proper transform on $S$. Then $\Gamma \sim 6 H-D$, where $D$ is an effective $\pi$-exceptional divisor on $S$. We write $D=D_{E}+D^{\prime}$, where $D_{E}$ is supported on $\operatorname{Supp}(E)$ and is contracted to $\operatorname{Irrat}(\Sigma) \cap C$, whereas $D^{\prime}$ is contracted to the Du Val singularities of $\Sigma$ situated on $C$. From $C \sim 6 H$ and $E \cdot H=E \cdot D^{\prime}=0$ we deduce

$$
-\kappa=-K_{S} \cdot \Gamma=E \cdot(6 H-D)=-E \cdot D=-E \cdot D_{E},
$$

i.e the presence of $\mathrm{Du}$ Val singularities does not affect $\kappa$ (this will be used in all cases discussed below). Thus, (26) reads

$$
v_{0}=\max \{g, g-\kappa-1\}=\max \left\{g, g-E \cdot D_{E}-1\right\} \leq 39 .
$$

Since $g \in\{14,15\}$, (26) follows once

$$
-E \cdot D_{E} \leq 25
$$

We will check inequality (29) case by case.

4.4.4. The normal cone case. This is case (iii) in Proposition 4.6, i.e., $\Sigma$ is the cone with vertex $\mathfrak{v}$ over a smooth quartic $Y \subset \mathbb{P}^{2}$. Then $S=\mathbb{P}\left(\mathcal{O}_{Y} \oplus \omega_{Y}\right)$. If $E_{0}$ is the section contracted by $\pi$ to $\mathfrak{v}$, then $E_{0}^{2}=-4, E=-K_{S}=2 E_{0}, H \cdot E_{0}=0$, and $D_{E}=E_{0}$ (remember the argument in 4.3 .3 , ( $\left.\ddagger\right)$ ). Thus $-E \cdot D_{E}=-2 E_{0}^{2}=8$ and so (29) holds.

Next we examine case (i) in Proposition 4.6 (see [16] for the cases considered below).

4.4.5. Normal rational quartics: Case (a). This case is described in [16, $\S 2.2 .1]$.

Let $X$ be a weak (or generalized) del Pezzo surface of degree 2, i.e., $-K_{X}$ is nef and big and $K_{X}^{2}=2$, see e.g., [12. Then $\operatorname{Bs}\left(\left|-K_{X}\right|\right)=\varnothing, \operatorname{dim}\left(\left|-K_{X}\right|\right)=2$, and $\varphi_{\left|-K_{X}\right|}: X \rightarrow \mathbb{P}^{2}$ is generically finite, of degree 2 (see [16, p. 944]). A general member $B \in\left|-2 K_{X}\right|$ is a smooth, non-hyperelliptic curve of genus 3 (see [16, Lemma 2.1]). If $G \in\left|-K_{X}\right|$, then $B \cdot G=4$. Thus the triplet $(X, B, G)$ satisfies condition $\mathcal{C}_{4}$ and we can consider its separation $(S, H, E)$ as in (27), which is a basic triplet. One has $H^{2}=4$ and $-K_{S} \equiv E$, with $E^{2}=-2$. Note that $\left|\rho^{*}(G)\right|$ has dimension 2 and is base point free. Furthermore, $H \cdot \rho^{*}(G)=4$.

According to Theorem 4.8, $S$ is the minimal desingularization of the normal quartic surface $\Sigma:=\varphi_{|H|}(S) \subset$ $\mathbb{P}^{3}$, and $\pi=\varphi_{|H|}$ contracts $E$ (and no other curve) to a unique irrational singular point $p \in \Sigma$, with $p_{g}(p)=1$ (see Proposition 4.5 4 (ii-1)).

Let $C \sim 6 H$ be an irreducible curve on $\Sigma$ of geometric genus $g$, and let $\Gamma$ be the proper transform of $C$ on $S$. We have $\Gamma \equiv 6 H-D$, with $D=D_{E}+D^{\prime}$ as above. From (27),$-\kappa=E \cdot \Gamma=\left(\rho^{*}(G)-\Delta\right) \cdot \Gamma$. Since $\Gamma$ is irreducible and non-rational, we have $\Delta \cdot \Gamma \geqslant 0$. Hence $E \cdot \Gamma \leqslant\left(\rho^{*}(G)-\Delta\right) \cdot \Gamma \leqslant \rho^{*}(G) \cdot \Gamma=\rho^{*}(G) \cdot(6 H-D)$. Since $\rho^{*}(G)$ is nef, one has $\rho^{*}(G) \cdot D \geqslant 0$. Thus $-\kappa=E \cdot \Gamma=\rho^{*}(G) \cdot(6 H-D) \leqslant \rho^{*}(G) \cdot(6 H)=24$. This proves (29).

Remark 4.9. An equivalent description of $\Sigma$ is gotten by taking the image of $\mathbb{P}^{2}$ via the rational map determined by a linear system of curves of degree 6 with 7 double and 4 simple base points all on a cubic.

4.4.6. Normal rational quartics: Case (b). This case is described in [16, § 2.2.2].

Let $Z$ be a weak del Pezzo surface of degree 1. Then $\left|-K_{Z}\right|$ is a pencil, and $\operatorname{Bs}\left(\left|-K_{Z}\right|\right)$ consists of a single point $b$ (cf. [16, p. 944]). If $L \in\left|-K_{Z}\right|$, then $b$ is a smooth point of $L$. Let $L^{\prime}$ be the irreducible component of $L$ containing $b$. One can choose a point $q \in L^{\prime}$ such that:

(1) $q$ is a smooth point for $L$, and

(2) $\mathcal{O}_{L}(b-q), \mathcal{O}_{L}(2 b-2 q)$ are not isomorphic to $\mathcal{O}_{L}$.

Then there exists a unique point $q_{1} \in L^{\prime}$ satisfying $\mathcal{O}_{L}\left(q_{1}\right) \cong \mathcal{O}_{L}(3 b-2 q)$ and $q_{1} \neq b$ by condition (2). 
Let $f: X \rightarrow Z$ be the blowup of $Z$ at $q$ with exceptional divisor $\Xi$, and let $G$ be the proper transform of $L$ on $X$. Let $b^{\prime}=f^{-1}(b)$ and $\left\{q^{\prime}\right\}=G \cap \Xi$. The points $b^{\prime}$ and $q^{\prime}$ are contained in the proper transform $G^{\prime}$ of $L^{\prime}$. There is a smooth point $q_{1}^{\prime} \in G$ such that $\mathcal{O}_{G}\left(3 b^{\prime}-2 q^{\prime}\right) \cong \mathcal{O}_{G}\left(q_{1}^{\prime}\right)$. Then $f\left(q_{1}^{\prime}\right)=q_{1},\left\{q_{1}^{\prime}\right\}=\operatorname{Bs}(|3 G+\Xi|)$ and $h^{0}\left(X, \mathcal{O}_{X}(3 G+\Xi)\right)=4$.

A general member $B \in|3 G+\Xi|$ is a smooth, non-hyperelliptic curve of genus 3 , and $(X, B, G)$ is a triplet satisfying condition $\mathcal{C}_{1}$, see [16, Lemma 2.2]. The separation of $B$ and $G$ consists in blowing-up $\rho: S \rightarrow X$ at $q_{1}^{\prime}$ with exceptional divisor $\Delta$. Letting $H, E$, and $\Xi^{\prime}$ be the proper transforms of $B, G$ and $\Xi$, respectively, we get a basic triplet $(S, H, E)$ with $H \cdot E=0, H^{2}=4, K_{S}=-E$, and $E^{2}=-1$.

We let $\Lambda$ be the total transform of $L$ on $S$. Then $\Lambda$ is nef, $|\Lambda|$ is a pencil with $\Lambda^{2}=1$, with a single base point, $\Lambda \cdot H=4$, and $E=\Lambda-\Xi^{\prime}-\Delta$, where $\Lambda \cdot \Xi^{\prime}=\Lambda \cdot \Delta=0$.

One has $\operatorname{Bs}(|H|)=\varnothing$, and $\pi=\varphi_{|H|}: S \rightarrow \mathbb{P}^{3}$ is the minimal desingularization of the quartic $\Sigma=\pi(S)$, which contracts $E$ (and only this curve) to an irrational singular point $p \in \operatorname{Irrat}(\Sigma)$ with $p_{g}(p)=1$.

For an irreducible curve $C \sim 6 H$ on $\Sigma$ of geometric genus $g \in\{14,15\}$, we let as before $\Gamma$ be the proper transform of $C$ on $S$. Then $\Gamma \equiv 6 H-D$, with $D=D_{E}+D^{\prime}$ (cf. (28) $)$. Since $\Gamma$ is non-rational, we have $\Xi^{\prime} \cdot \Gamma \geqslant 0$ and $\Delta \cdot \Gamma \geqslant 0$. Furthermore, $\Lambda \cdot D \geqslant 0$ since $\Lambda$ is nef. Hence

$$
-\kappa=E \cdot \Gamma=\left(\Lambda-\Xi^{\prime}-\Delta\right) \cdot \Gamma \leqslant \Lambda \cdot \Gamma=\Lambda \cdot(6 H-D) \leqslant 6 \Lambda \cdot H=24 .
$$

Thus again (29) is satisfied.

Remark 4.10. The quartic $\Sigma \subset \mathbb{P}^{3}$ in this case is the image of $\mathbb{P}^{2}$ under the rational map determined by a linear system of curves of degree 9 with 8 triple and one double base points, all on a cubic.

\subsubsection{Normal rational quartics: Case (c). This case is described in [16, $\S 2.2 .3]$.}

Consider the Hirzebruch surface $\mathbb{F}_{1}=\mathbb{P}\left(\mathcal{O}_{\mathbb{P}^{1}} \oplus \mathcal{O}_{\mathbb{P}^{1}}(1)\right)$. Let $\Xi$ be the section with $\Xi^{2}=-1$ and $F$ a ruling. Fix a point $x_{0} \in \Xi$, and let $F_{0}$ be the ruling containing $x_{0}$. There exists a reduced divisor $\Delta \in|2 \Xi+6 F|$ such that:

(1) if $f: V \rightarrow \mathbb{F}_{1}$ is the double covering branched along $\Delta$, then $V$ has only Du Val singularities;

(2) $\Xi \not \Delta ;$

(3) $x_{0} \in \Delta \cap \Xi$ and $\operatorname{mult}_{x_{0}}\left(\left.\Delta\right|_{\Xi}\right)=1$;

(4) $F_{0} \not \subset$ and $F_{0} \cap \Delta=\left\{x_{0}\right\}$.

Fixing such a $\Delta$, we let $\lambda: Y \rightarrow V$ be the minimal desingularization of the double covering $V$ as in (1). The surface $Y$ is rational, because it carries the pencil of rational curves $\left|\widetilde{\lambda}^{*}(F)\right|$, where $\widetilde{\lambda}=f \circ \lambda: Y \rightarrow \mathbb{F}_{1}$. One has $K_{Y} \sim \widetilde{\lambda}^{*}\left(K_{\mathbb{F}_{1}}+\Xi+3 F\right) \sim \widetilde{\lambda}^{*}(-\Xi)$. Letting $G:=\widetilde{\lambda}^{*}(\Xi)$, by (2) and (3) above, there exists an irreducible component $G^{\prime} \subset G$ such that the induced morphism $G^{\prime} \rightarrow \Xi$ is a double covering, whereas the other components of $G$ are contracted by $\widetilde{\lambda}$ to points of $\Xi$. These components are also contracted to singular points of $V$, hence by (1), they are rational curves with self-intersection -2 .

The morphism $\tilde{\lambda}$ is finite over an open neighborhood of $x_{0}$ and $\widetilde{\lambda}^{-1}\left(x_{0}\right)$ consist of a single point $b^{\prime} \in G^{\prime}$. One has $\widetilde{\lambda}^{*}\left(F_{0}\right)=F_{1}+F_{2}$, where $F_{1}, F_{2}$ are $(-1)$-curves such that $F_{1} \cdot F_{2}=1$ and $F_{1} \cap F_{2}=\left\{b^{\prime}\right\}$.

Let $\mu: Y \rightarrow S$ be the blowdown of $F_{1}$. Letting

$$
E:=\mu_{*}(G) \sim-K_{S}, \quad L:=\mu_{*}\left(F_{2}\right), \text { and } b:=\mu\left(b^{\prime}\right),
$$

we get $\mu^{*}(E)=G+F_{1}$ and $\widetilde{\lambda}^{*}(F) \sim \mu^{*}(L)$. By [16, Lemma 2.5], $|L+2 E|$ is base point free and its general member $H$ is a smooth, non-hyperelliptic curve of genus 3, with $H^{2}=4$. Then $\pi=\varphi_{|H|}$ maps $S$ to a normal quartic $\Sigma \subset \mathbb{P}^{3}$ and contracts $E$ (and only $E$ ) to a unique irrational singular point $p \in \operatorname{Irrat}(\Sigma)$ with $p_{g}(p)=1$.

Note that $E$ contains a component $E^{\prime}:=\mu_{*}\left(G^{\prime}\right)$. The other (possible) components of $E$ are rational curves with self-intersection -2 and so, these have zero intersection with $K_{S} \equiv-E$. Hence $-1=E^{2}=E \cdot E^{\prime}$. Since $L=\mu_{*}\left(F_{2}\right)$, where $L^{2}=0$, the linear system $|L|$ is a base point free pencil of rational curves. One has $L \cdot E=L \cdot E^{\prime}=2$ (i.e., $L$ has zero intersection with the components of $E$ different from $E^{\prime}$ ), and so $L \cdot H=4$. 
For $C \sim 6 H$ on $\Sigma$ of geometric genus $g$, we have $\Gamma \equiv 6 H-D$, with $D=D_{E}+D^{\prime}$. Let $\alpha$ be the multiplicity of $E^{\prime}$ in $D_{E}$. Then, from (28), $\kappa=E \cdot D_{E}=\alpha E \cdot E^{\prime}=-\alpha$. On the other hand, $2 \leqslant L \cdot \Gamma=24-2 \alpha$, thus $-\kappa=\alpha \leqslant 11$ and so, (29) holds.

4.4.8. Quartic monoids. This case corresponds to quartic surfaces with a triple point (see [16, $\S 2.4]$ ).

Explicitly, let $B$ be a smooth quartic in $\mathbb{P}^{2}$ and let $G$ be a cubic in $\mathbb{P}^{2}$, not necessarily reduced or irreducible. Performing the separation of $\left(\mathbb{P}^{2}, B, G\right)$, we have a basic triplet $(S, H, E)$ with $E^{2}=-3$ and $K_{S} \sim-E$. The morphism $\pi=\varphi_{|H|}$ sends $S$ to a normal quartic $\Sigma \subset \mathbb{P}^{3}$, contracting $E$ to the only irrational (triple) point $p$ of $\Sigma$, with $p_{g}(p)=1$.

Letting $\Lambda=H-E \sim H+K_{S}$, we obtain a base point free linear system $|\Lambda|$ of dimension 2 , with $\Lambda^{2}=1$ and $p_{a}(\Lambda)=0$. The morphism $\varphi_{|\Lambda|}: S \rightarrow \mathbb{P}^{2}$ factors through the (stereographic) projection $\Sigma \rightarrow \mathbb{P}^{2}$ with center $p$. In fact, this morphism is nothing but the above separation.

For $C \sim 6 H$ on $\Sigma$ of geometric genus $g$ we have, as usual, $\Gamma \equiv 6 H-D$, with $D=D_{E}+D^{\prime}$. Since $|\Lambda|$ cuts out on $\Gamma$ a linear series of dimension 2 , we have $4 \leqslant \Lambda \cdot \Gamma=(H-E) \cdot(6 H-D)=24+E \cdot D_{E}$. Hence, from (28), $-\kappa=-E \cdot D_{E} \leqslant 20$ so (29) holds.

Next we turn to case (ii) in Proposition 4.6. In [16, § 2.3] (cf. also [24]) there is a classification, which we will go through. From Proposition 4.5 - $(i i)$, the cardinality of $\operatorname{Irrat}(\Sigma)$ can be either 1 or 2.

4.4.9. Ruled elliptic normal quartics: Case (a). This case is described in [16, $\S 2.3 .2]$.

Let $Y$ be a smooth, elliptic curve, and let $q_{1}, q_{2} \in Y$ be such that $2 q_{1} \uparrow 2 q_{2}$. Letting $\mathcal{E}=\mathcal{O}_{Y}\left(q_{1}\right) \oplus \mathcal{O}_{Y}\left(q_{2}\right)$ and $X=\mathbb{P}(\mathcal{E})$, we consider the structure morphism $\sigma: X \rightarrow Y$ and the fibres $F_{i}:=\sigma^{*}\left(q_{i}\right), i=1,2$. Let further $H_{\mathcal{E}}$ be the tautological divisor class on $X$ and $F$ the numerical class of a fibre.

The surface $X$ possesses two sections $\Xi_{i} \sim H_{\mathcal{E}}-F_{i}$, for $i=1,2$. One has $\Xi_{1} \cap \Xi_{2}=\varnothing$ and $\left|-K_{X}\right|=\{G\}$, where $G:=\Xi_{1}+\Xi_{2}$. A general member $B \in\left|H_{\mathcal{E}}-K_{X}\right|$ is a smooth, non-hyperelliptic curve of genus 3 which intersects transversally $\Xi_{i}$ at a point $x_{i}, i=1,2$.

Let $\rho: S \rightarrow X$ be the blowup of $X$ at the points $x_{i}$ with exceptional divisors $\Delta_{i}$, for $i=1,2$. Consider the proper transform $\Xi_{i}^{\prime}$ of $\Xi_{i}$ for $i=1,2$, the divisor $E=\Xi_{1}^{\prime}+\Xi_{2}^{\prime} \equiv-K_{S}$, and the proper transform $H$ of $B$ on $S$. Then $K_{S}^{2}=-2, H^{2}=4, \Xi_{i}^{\prime 2}=-1$, and $H \cdot \Xi_{i}^{\prime}=0$, for $i=1,2$. We abuse notation and denote by $F$ the total transform on $S$ of a ruling of $X$. One has $H \cdot F=3$.

Thus we got a separation $(S, H, E)$ of $B$ and $G$. The linear system $|H|$ on $S$ is base point free of dimension 3 , and $\pi=\varphi_{|H|}$ maps $S$ to a quartic surface $\Sigma$ in $\mathbb{P}^{3}$ with $\operatorname{Irrat}(\Sigma)=\left\{p_{1}, p_{2}\right\}$, where $p_{i}=\pi\left(\Xi_{i}^{\prime}\right)$ is a simple elliptic singularity with $p_{g}\left(p_{i}\right)=1$, for $i=1,2$. Since $H \cdot F=3, \Sigma$ is swept out by an elliptic pencil of rational normal cubics.

Take $C \sim 6 H$ on $\Sigma$ of geometric genus $g$. Then $\Gamma \equiv 6 H-D$, with $D_{E}=\alpha_{1} \Xi_{1}^{\prime}+\alpha_{2} \Xi_{2}^{\prime}, \alpha_{1}, \alpha_{2}$ non-negative integers. From (28), one has $-\kappa=\alpha_{1}+\alpha_{2}$. On the other hand, $\Gamma \cdot F=18-\left(\alpha_{1}+\alpha_{2}\right) \geqslant 2$, because $g>1$. Hence $-\kappa \leqslant 16$ and so, (29) holds.

4.4.10. Ruled elliptic normal quartics: Case (b). This case is contained in [16, $\S 2.3 .1$ ], to which we refer for details.

Let $Y$ be a smooth, elliptic curve with a line bundle $A$ of degree 2. Letting $\mathcal{E}=\mathcal{O}_{Y} \oplus A$ we consider the elliptic ruled surface $X=\mathbb{P}\left(\mathcal{O}_{Y} \oplus A\right)$ with the structure morphism $\sigma: X \rightarrow Y$. We let $\Xi_{1}$ denote the unique section of $\sigma$ with $\Xi_{1}^{2}=-2, H_{\mathcal{E}}$ the tautological line bundle, and $F$ the numerical class of a ruling. One has $h^{0}\left(X, \mathcal{O}_{X}\left(H_{\mathcal{E}}\right)\right)=3$ and $H_{\mathcal{E}}^{2}=2, H_{\mathcal{E}} \cdot \Xi_{1}=0$. Furthermore $-K_{X} \equiv H_{\mathcal{E}}+\Xi_{1}$. Hence $\left|-K_{X}\right|$ has $\Xi_{1}$ as a fixed component and $\left|H_{\mathcal{E}}\right|$ as its movable part. If $G \in\left|-K_{X}\right|$, then $h^{0}\left(G, \mathcal{O}_{G}\right)=2$. Letting $G=\Xi_{1}+\Xi_{2}$, we note that either $\Xi_{1} \cap \Xi_{2}=\varnothing$, or $\Xi_{2}$ consists of $\Xi_{1}$ plus the sum of two fibres with class in $\left|\sigma^{*}(A)\right|$.

A general member $B \in\left|-K_{X}+\sigma^{*}(A)\right|$ is a smooth, non-hyperelliptic curve of genus 3 with $B^{2}=8$ and $B \cdot G=4$. Note that $B \cdot \Xi_{1}=0$, so $B \cap \Xi_{1}=\varnothing$. Thus $(X, B, G)$ is a $\mathcal{C}_{4}$-triplet.

Performing a separation of $(X, B, G)$, we obtain a basic triplet $(S, H, E)$ with $H$ and $E \sim-K_{S}$ being the proper transforms of $B$ and $G$, respectively. We let $E_{i}$ denote the proper transforms of $\Xi_{i}$, for $i=1,2$. One has $K_{S}^{2}=-4, H^{2}=4, H \cdot E_{i}=0$, and $E_{i}{ }^{2}=-2$, for $i=1,2$. We abuse notation and let $F$ still denote the total transform of a ruling $F$ on $S$. One has $H \cdot F=2$. 
The linear system $|H|$ is base point free of dimension 3, and $\pi=\varphi_{|H|}$ maps $S$ to a normal quartic $\Sigma \subset \mathbb{P}^{3}$. Since $H \cdot F=2$, the surface $\Sigma$ is swept out by an elliptic pencil of conics $|F|$. Furthermore, $E=E_{1}+E_{2}$ is contracted to one or two irrational singular points. More precisely, if $\Xi_{1} \cap \Xi_{2}=\varnothing$ and so $E_{1} \cap E_{2}=\varnothing$, then $p_{i}=\pi\left(E_{i}\right), i=1,2$, are two distinct simple elliptic singularities of $\Sigma$ with $p_{g}\left(p_{i}\right)=1$. Otherwise $E_{2}=E_{1}+F_{1}+F_{2}$, where $F_{1}, F_{2}$ are two (may be coinciding) (-2)-curves obtained as a result of two blowups on each of two (may be coinciding) fibres in $\left|\sigma^{*}(A)\right|$. In this case $\Sigma$ has a unique irrational singular point $p$ with $p_{g}(p)=2$ (see Proposition 4.5 - $(i i-2)$ ).

Take a curve $C \sim 6 H$ on $\Sigma$ of geometric genus $g$. According to the cardinality of $\operatorname{Irrat}(\Sigma)$, we consider the following cases.

(1) If $\operatorname{Irrat}(\Sigma)=\left\{p_{1}, p_{2}\right\}$ with $p_{1} \neq p_{2}$, then $\Gamma \equiv 6 H-D$ and $D_{E}=\alpha_{1} E_{1}+\alpha_{2} E_{2}$ with $\alpha_{i}$ non-negative integers. One has $-\kappa=2\left(\alpha_{1}+\alpha_{2}\right)$. On the other hand $F \cdot \Gamma=12-\left(\alpha_{1}+\alpha_{2}\right) \geqslant 2$ since $g>1$ thus, from $(28),-\kappa \leqslant 20$.

(2) If Irrat $(\Sigma)=\{p\}$, then $\Gamma \equiv 6 H-D$, with $D_{E}=\alpha E_{1}+\beta_{1} F_{1}+\beta_{2} F_{2}$ for some non-negative integers $\alpha, \beta_{1}, \beta_{2}$. One has $K_{S} \cdot F_{1}=K_{S} \cdot F_{2}=0$, thus $-\kappa=2 \alpha$. As before, $F \cdot \Gamma=12-\alpha \geqslant 2$, hence again $-\kappa \leqslant 20$.

In any case, (29) holds.

4.4.11. Ruled elliptic normal quartics: Case (c). This case is treated in [16, $\S 2.3 .2$, Case C2-2].

Let $Y$ be a smooth, irreducible elliptic curve, and let $q \in Y$. Taking $0 \neq \xi \in \operatorname{Ext}^{1}\left(\mathcal{O}_{Y}(q), \mathcal{O}_{Y}(q)\right)$ we consider the corresponding rank two vector bundle $\mathcal{E}:=\mathcal{E}_{\xi}$ on $Y$. We let $X:=\mathbb{P}(\mathcal{E})$ and $F_{q}:=\sigma^{*}(q)$, where $\sigma: X \rightarrow Y$ is the structure morphism. Let $H_{\mathcal{E}}$ be the tautological divisor class on $X$ and $F$ the numerical class of a fibre.

On $X$ we consider a section $\Xi_{0} \sim H_{\mathcal{E}}-F_{q}$ corresponding to $\mathcal{E} \rightarrow \mathcal{O}_{Y}(q)$, so that $\Xi_{0}^{2}=0$. Notice that $\left|\Xi_{0}\right|=\left\{\Xi_{0}\right\}$, as it follows from $h^{0}(Y, \mathcal{E}(-q))=1$. One has $\left|-K_{X}\right|=\{G\}$, where $G:=2 \Xi_{0}$.

Since $\left|H_{\mathcal{E}}-K_{X}\right|=\left|3 \Xi_{0}+F_{q}\right|$, one finds that $\operatorname{Bs}\left(\left|3 \Xi_{0}+F_{q}\right|\right)$ consists of the single point $b:=\Xi_{0} \cap F_{q}$. The general member $B \in\left|3 \Xi_{0}+F_{q}\right|$ is a smooth, non-hyperelliptic curve of genus 3 with $B \cdot \Xi_{0}=1$. Thus $(X, B, G)$ is a $\mathcal{C}_{2}$-triplet. The separation of $B$ and $G$ proceeds in two steps as follows.

On the first step, we let $\rho_{1}: X_{1} \rightarrow X$ be the blowup at $b$ with exceptional divisor $\Delta_{1}$, and let $G_{1}=$ $\rho_{1}^{*}(G)-\Delta_{1}, B_{1}=\rho_{1}^{*}(B)-\Delta_{1}, \Xi_{0}^{\prime}=\rho_{1}^{*}\left(\Xi_{0}\right)-\Delta_{1}$, and $F_{q}^{\prime}=\rho_{1}^{*}\left(F_{q}\right)-\Delta_{1}$. Since $B_{1} \cdot G_{1}=1$, we get a $\mathcal{C}_{1}$-triplet $\left(X_{1}, B_{1}, G_{1}\right)$ and a smooth point $b_{1}$ on $G_{1}$ with $\mathcal{O}_{G_{1}}\left(B_{1}\right) \cong \mathcal{O}_{G_{1}}\left(b_{1}\right)$. One has $b_{1} \in \Delta_{1}$, because $G_{1}=2 \Xi_{0}^{\prime}+\Delta_{1}$ and $B_{1} \cdot \Xi_{0}^{\prime}=0$, and furthermore, $b_{1} \in \operatorname{Bs}\left(\left|B_{1}\right|\right)$ and $b_{1} \notin F_{q}^{\prime}$.

On the second step, we consider the blowup $\rho_{2}: S \rightarrow X_{1}$ of $X_{1}$ at $b_{1}$ with exceptional divisor $\Delta_{2}$. In this way, we arrive at a basic triplet $(S, H, E)$, where

$$
H=B_{1}^{*}-\Delta_{2}=3 \Xi_{0}^{\prime \prime}+F_{q}^{\prime \prime}+3 \Delta_{1}^{\prime}+2 \Delta_{2} \text { and } E=G_{1}^{*}-\Delta_{2}=2 \Xi_{0}^{\prime \prime}+\Delta_{1}^{\prime} \sim-K_{S} .
$$

Here $\Xi_{0}^{\prime \prime}, F_{q}^{\prime \prime}$, and $\Delta_{1}^{\prime}$ are the proper transforms on $S$ of $\Xi_{0}^{\prime}, F_{q}^{\prime}$, and $\Delta_{1}$, respectively. One has $\Xi^{\prime 2}=$ $-1, \Delta_{1}^{\prime 2}=-2$, and $\Xi^{\prime} \cdot \Delta_{1}^{\prime}=1$. Abusing notation, we still denote by $F$ be total transform on $S$ of a ruling of $X$.

The linear system $|H|$ is base point free of dimension 3 , with $H^{2}=4$ and $H \cdot F=3$. The associated morphism $\pi=\varphi_{|H|}$ sends $S$ to a quartic surface $\Sigma$ in $\mathbb{P}^{3}$ with a unique irrational singular point $p=\pi(E)$. It is swept out by an elliptic pencil $|F|$ of rational normal cubics. One has $E^{2}=-2$ and $p_{g}(p)=2$.

For $C \sim 6 H$ we have $\Gamma \equiv 6 H-D$, with $D_{E}=\alpha \Xi^{\prime}+\beta \Delta_{1}^{\prime}$ for some non-negative integers $\alpha$ and $\beta$. Thus, from (28), $-\kappa=\alpha$. Since $\Gamma \cdot F=18-\alpha \geqslant 2$, then $-\kappa \leqslant 16$. Thus again (29) holds.

This ends the discussion of the normal cases. We turn next to the non-normal cases (see Proposition 4.7).

4.4.12. Non-normal genus 2 scrolls. In this case $\Sigma$ is a cone over an irreducible plane quartic $Y$ with a node or a cusp (see [25, Prop. 2.6]). The one-dimensional singular locus of $\Sigma$ is a double line $\ell$ passing through the vertex $\mathfrak{v}$ of $\Sigma$.

The minimal desingularization of $\Sigma$ is the surface $S=\mathbb{P}\left(\mathcal{O}_{G} \oplus L\right)$, where $G$ is the normalization of $Y$ and $L \in \operatorname{Pic}^{4}(G), L \neq \omega_{Y}^{\otimes 2}$. The morphism $\pi: S \rightarrow \Sigma \subset \mathbb{P}^{3}$ is determined by the tautological line bundle $\mathcal{O}_{S}(1)$. Letting $H$ and $E$, be the sections corresponding to $\mathcal{O}_{G} \oplus L \rightarrow L$ and to $\mathcal{O}_{G} \oplus L \rightarrow \mathcal{O}_{G}$, respectively, we 
obtain $H^{2}=-E^{2}=4, \mathcal{O}_{S}(1)=\mathcal{O}_{S}(H), H \equiv E+4 F$, and $K_{S} \equiv-2 E-2 F \equiv-2 H+6 F$, where $F$ stands, as usual, for the class of a ruling of $S$.

Let $C \sim 6 H$ be an irreducible curve of geometric genus $g \in\{14,15\}$ on $\Sigma$, and $\Gamma$ its proper transform on $S$. Since $C$ is cut out on $\Sigma$ by a smooth surface of degree 6 , we have $\Gamma \equiv 6 H-\alpha E$, where $\alpha \in\{0,1\}$. Thus $-\kappa=-K_{S} \cdot \Gamma=12+6 \alpha \leq 18$, and so (29) holds.

Next we consider the non-normal elliptic scrolls, see Proposition 4.7. There are two types of such scrolls described in [25, § 1] as cases (II-1) and (II-2).

4.4.13. Non-normal elliptic scrolls: case (a). Take a smooth, irreducible elliptic curve $G$. Let $N \in \operatorname{Pic}^{0}(G)$ be non-trivial, and let $M \in \operatorname{Pic}^{2}(G)$. Consider the ruled surface $S:=\mathbb{P}\left(\mathcal{O}_{G} \oplus N\right)$ together with the structure morphism $\sigma: S \rightarrow G$. Let $D_{1}$ and $D_{2}$ be the sections associated with $\mathcal{O}_{G} \oplus N \rightarrow \mathcal{O}_{G}$ and $\mathcal{O}_{G} \oplus N \rightarrow N$, respectively, and let $F$ be the ruling class. Then $K_{S} \equiv-2 D_{1}$, and the line bundle $H:=\mathcal{O}_{S}\left(D_{1}\right) \otimes \sigma^{*}(M)$ induces a finite birational morphism $\pi=\varphi_{|H|}: S \rightarrow \mathbb{P}^{3}$ onto an irreducible quartic surface $\Sigma$.

The images $\ell_{1}=\pi\left(D_{1}\right)$ and $\ell_{2}=\pi\left(D_{2}\right)$ are skew double lines of $\Sigma$, and $\operatorname{Sing}(\Sigma)=\ell_{1} \cup \ell_{2}$. The image under $\pi$ of any fibre of $S$ is a line meeting both $\ell_{1}$ and $\ell_{2}$. The general plane section of $\Sigma$ has two nodes at the intersection points with $\ell_{1}$ and $\ell_{2}$, and its normalizations is $G$.

Let $C \sim 6 H$ be an irreducible curve of geometric genus $g$ on $\Sigma$. Then $\Gamma \sim 6 H \equiv 6 D_{1}+12 F$, and so, $-\kappa=-K_{S} \cdot \Gamma=24$, proving again (29).

Remark 4.11. The construction of this scroll is classical. Once skew lines $\ell_{1}, \ell_{2}$ in $\mathbb{P}^{3}$ have been fixed, take a smooth, irreducible, elliptic curve $G$ and two degree two maps $f_{i}: G \rightarrow \ell_{i}, i=1,2$. For each $x \in G$, let $\ell_{x}=\left\langle f_{1}(x), f_{2}(x)\right\rangle$. Then $\Sigma=\cup_{x \in G} \ell_{x}$.

4.4.14. Non-normal elliptic scrolls: case (b). Let $G$ be a smooth, irreducible elliptic curve, and let $M \in$ $\operatorname{Pic}^{2}(G)$ be a line bundle on $G$. Let $\mathcal{E}:=\mathcal{E}_{\xi}$ be the rank-two vector bundle on $G$ fitting in the non-split sequence

$$
0 \rightarrow \mathcal{O}_{G} \rightarrow \mathcal{E} \rightarrow \mathcal{O}_{G} \rightarrow 0
$$

associated to the choice of a non-zero $\xi \in \operatorname{Ext}^{1}\left(\mathcal{O}_{G}, \mathcal{O}_{G}\right)$. Let $S:=\mathbb{P}(\mathcal{E})$, together with the structure morphism $\sigma: S \rightarrow G$ and the fibre class $F$. Let $D_{1}$ be the section corresponding to $\mathcal{E} \rightarrow \mathcal{O}_{G}$, and let $H:=\mathcal{O}_{S}\left(D_{1}\right) \otimes \sigma^{*}(M)$. As in $\S$ 4.4.13, $H$ defines a finite morphism $\pi=\varphi_{|H|}: S \rightarrow \mathbb{P}^{3}$ onto an irreducible quartic surface $\Sigma$. Then $\pi\left(D_{1}\right)$ is a double line $\ell$ of $\Sigma$, and any fibre of $S$ is sent via $\pi$ to a line of $\Sigma$ crossing $\ell$. One has $\operatorname{Sing}(\Sigma)=\ell$. The general plane section $H$ of $\Sigma$ has an $A_{3}$-singularity at $H \cap \ell$, and its normalizations is $G$. Since $\Gamma \sim 6 H \equiv 6 D_{1}+12 F$ and $K_{S} \equiv-2 D_{1}$, the computations go as in $\S 4.4 .13$ proving (29).

According to Proposition 4.7, we are left with the rational case. This gives rise to three items (see (III-A), (III-B), (III-C) in [25, § 1]).

4.4.15. Rational non-normal quartics: case (a), the Segre surface. The Segre surface $\Sigma \subset \mathbb{P}^{3}$ is the image of a normal surface $\widehat{\Sigma} \subset \mathbb{P}^{4}$ of degree 4 with at most Du Val singularities under the linear projection $\Pi_{p}: \mathbb{P}^{4} \rightarrow \mathbb{P}^{3}$ with center $p \notin \widehat{\Sigma}$. The surface $\widehat{\Sigma}$ is the anticanonical image of a weak del Pezzo surface of degree 4 , i.e., the blowup of $\mathbb{P}^{2}$ at 5 points, see [21].

Apart from its one-dimensional singular locus $\Lambda$, which is in general a double conic (see Remark in [25, p. 277] for details), $\Sigma$ can have further isolated Du Val singularities off $\Lambda$. We have $-\kappa=-K_{S} \cdot \Gamma=6 K_{S}^{2}=24$, proving (29).

4.4.16. Rational non-normal quartics: case (b). In this case $\Sigma$ has a singular line $\ell$, and its general plane section has geometric genus 2 , hence it is either nodal or cuspidal. Besides, $\Sigma$ may have isolated Du Val singularities off $\ell$ (cf. [25, Case (III-C), p. 269]).

The surface $S$ is obtained by successively blowing-up $\mathbb{P}^{2}$ at 9 points. The morphism $\pi: S \rightarrow \Sigma \subset \mathbb{P}^{3}$ is defined by $H \sim 4 L-2 E_{1}-\sum_{i=2}^{9} E_{i}$, where $L$ is the proper transform of a line in $\mathbb{P}^{2}$ and $E_{i}$, for $i=1, \ldots, 9$, are the (total) exceptional divisors of the blowups. In particular, $K_{S}=-3 L+\sum_{i=1}^{9} E_{i}$, and $h^{0}\left(S, \mathcal{O}_{S}\left(-K_{S}\right)\right)=1$, i.e., there is only one cubic curve on $\mathbb{P}^{2}$ passing through the 9 blown-up points, 
corresponding to a unique effective anticanonical divisor $D$ on $S$. Note that $\pi(D)=\ell$ and that $\Sigma$ is swept out by a pencil of conics cut out by the planes containing $\ell$, with the pullback $\Lambda \sim L-E_{1}$ on $S$.

The surface $\Sigma$ can have further singularities along $\ell$ described in [25, Case (III-C), p. 269]. Consider the morphisms $S \stackrel{\rho}{\rightarrow} \widehat{\Sigma} \stackrel{\nu}{\rightarrow} \Sigma$, where $\nu$ is the normalization, $\rho$ is the minimal resolution of singularities of $\widehat{\Sigma}$, and $\pi=\nu \circ \rho$. Then any singular point of $\widehat{\Sigma}$ which is not Du Val lies on $\nu^{-1}(\ell)$ and is a rational triple point. The number of such triple points is at most 2, their images on $\Sigma$ are also triple points for $\Sigma$ (which in this case is a monoid). If $\Delta$ is the fundamental cycle of such a triple point, then $\Delta<D$. Moreover $\Lambda \cdot \Delta=1$. Let $A$ be an irreducible component of $\Delta$. Since $H=\Lambda-K_{S}$ and $H \cdot A=0$, we have $K_{S} \cdot A=\Lambda \cdot A \in\{0,1\}$. Since $A \cong \mathbb{P}^{1}$, then $A^{2} \in\{-2,-3\}$.

For two possible rational triple points of $\Sigma$, we let $\Delta_{1}, \Delta_{2}$ denote their fundamental cycles on $S$. If $C \sim 6 H$ is an irreducible curve of geometric genus $g$ on $\Sigma$ with the proper transform $\Gamma$ on $S$, then $\Gamma \equiv$ $6 H-\Delta_{1}^{\prime}-\Delta_{2}^{\prime}$, where the support of $\Delta_{i}^{\prime}$ is contained in the support of $\Delta_{i}$, for $i=1,2$. If $A_{i}$ is the unique component of $\Delta_{i}$ such that $\Lambda \cdot A_{i}=1$, we let $\alpha_{i}$ be the multiplicity of $A_{i}$ in $\Delta_{i}^{\prime}$, for $i=1,2$. Thus, $-\kappa=-K_{S} \cdot \Gamma=12+K_{S} \cdot\left(\Delta_{1}^{\prime}+\Delta_{2}^{\prime}\right)=12+\left(\alpha_{1}+\alpha_{2}\right)$. On the other hand, since $\Gamma$ has genus $g \geqslant 14$, we have $2 \leqslant \Gamma \cdot \Lambda=12-\left(\alpha_{1}+\alpha_{2}\right)$. Hence $-\kappa \leqslant 22$, and so, (29) holds.

4.4.17. Rational non-normal quartics: case (c). In this case $\Sigma$ is the image of a smooth surface $S \subset \mathbb{P}^{5}$ via the linear projection $\Pi_{\ell}: \mathbb{P}^{5} \rightarrow \mathbb{P}^{3}$ with center a line $\ell \subset \mathbb{P}^{5}$ disjoint from $S$ (cf. [25, Case (III-A)]). In this situation, $\pi=\Pi_{\ell \mid S}: S \rightarrow \Sigma$ is the minimal desingularization of $\Sigma$, and either:

(1) $S$ is the Veronese surface, i.e., $S \cong \mathbb{P}^{2}$ embedded in $\mathbb{P}^{5}$ via the linear system $|2 L|$, where $L$ is a line on $\mathbb{P}^{2}$, and $\Sigma$ is the Steiner's Roman surface, or

(2) $S \cong \mathbb{F}_{0}$, and its embedding in $\mathbb{P}^{5}$ is given by $\left|F_{1}+2 F_{2}\right|$, where $F_{1}$ and $F_{2}$ are the two distinct rulings, or

(3) $S=\mathbb{F}_{2}$, and its embedding in $\mathbb{P}^{5}$ is given by $|D+F|$, where $D$ is a section with $D^{2}=2$, and $F$ is the ruling.

Let $C \sim 6 H$ on $\Sigma$ and $\Gamma$ on $S$ be as before. Then in all cases $-\kappa=36$, hence (29) does not hold. Therefore we have to directly check if (22) holds. Since $\Phi(4,6)=73$, (22) holds if $\operatorname{dim}(\mathcal{F})<23$. To see that this is the case, consider the Rohn exact sequence

$$
0 \rightarrow \mathcal{O}_{S}(1)^{\oplus 2} \rightarrow N_{S \mid \mathbb{P}^{5}} \rightarrow N_{\pi} \rightarrow 0
$$

(see [8, (2.2)]), where $N_{\pi}$ is the normal sheaf to the map $\pi$. Since $h^{1}\left(S, \mathcal{O}_{S}(1)\right)=0$ in the above three cases, one has

$$
\operatorname{dim} \mathcal{F} \leqslant h^{0}\left(S, N_{\pi}\right)=h^{0}\left(S, N_{S \mid \mathbb{P}^{5}}\right)-12 .
$$

On the other hand, $h^{0}\left(S, N_{S \mid \mathbb{P}^{5}}\right)$ is the dimension of the component of Hilbert scheme described by the surfaces $S$ in the cases (1)-(3). Notice that the surfaces in case (3) are specializations of the ones in case (2). Finally, we obtain

$$
h^{0}\left(S, N_{S \mid \mathbb{P}^{5}}\right)=\operatorname{dim}\left(\operatorname{Aut}\left(\mathbb{P}^{5}\right)\right)-\operatorname{dim}(\operatorname{Aut}(S))= \begin{cases}27 & \text { in case }(1) \\ 29 & \text { in case }(2) .\end{cases}
$$

(we leave the details to the reader; alternatively, see the proof of $[8$, Lemma $(2.3)]$ ). In conclusion, $\operatorname{dim}(\mathcal{F}) \leqslant$ $17<23$, which finishes our proof.

\section{REFERENCES}

[1] M. C. Beltrametti, A. J. Sommese, The adjunction theory of complex projective varieties, Exposition in Mathematics, 16 (1995), De Gruyter.

[2] J.W. Bruce, A stratification of the space of cubic surfaces, Math. Proc. Camb. Phil. Soc., 87 (1980), $427-441$.

[3] J. W. Bruce, T. C. Wall, On the classification of cubic surfaces, J. London Math. Soc., 19 (1979), 427-441.

[4] A. Cayley, A memoir on cubic surfaces, Phil. Trans. Roy. Soc., 159 (1869), 231-326.

[5] L. Chiantini, C. Ciliberto, On the Severi varieties of surfaces in $\mathbb{P}^{3}$, J. Alg. Geom., 8 (1999), 67-83.

[6] L. Chiantini, A. F. Lopez, Focal loci of families and the genus of curves on surfaces, Proc. Amer. Math. Soc., 127 (1999), 3451-3459. 
[7] L. Chiantini, A. F. Lopez, Z. Ran, Subvarieties of generic hypersurfaces in any variety, Mathematical Proceedings of the Cambridge Philosophical Society, 130 (2001), 259-268.

[8] C. Ciliberto, On the Hilbert Scheme of Curves of Maximal Genus in a Projective Space, Mathematische Zeitschrift, 194 (1987), 351-363.

[9] C. Ciliberto, M. Zaidenberg, Scrolls and hyperbolicity, Int. J. Math., 24 (2013), (4), 38-63.

[10] H. Clemens, Curves on generic hypersurfaces, Ann. Sci. École Norm. Sup., (4) 199 (1986), 629-636.

[11] A. I. Degtyarev, Classification of surfaces of degree four having a nonsimple singular point, Izv. Akad. Nauk SSSR, $\mathbf{5 3}$ (1989), 1269-1290; English transl. Math. USSR Izvestiya, 35 (1990), 607-627.

[12] I. V. Dolgachev, Classical algebraic geometry, Cambridge University Press, Cambridge, 2012.

[13] Ph. Griffiths, J. Harris, On the Noether-Lefschetz theorem and some remarks on codimension-two cycles, Math. Ann., 27 (1985), 31-51.

[14] J. Harris, J. Morrison, Moduli of curves, Graduate Texts in Mathematics, No. 187. Springer-Verlag, New York-Heidelberg, 1998.

[15] R. Hartshorne, Algebraic geometry, Graduate Texts in Mathematics, No. 52. Springer-Verlag, New York-Heidelberg, 1977.

[16] Y. Ishii, N. Nakayama, Classification of normal quartic surfaces with irrational singularities, J. Math. Soc. Japan, 56 (2004), (3), 941-965.

[17] E. J. N. Looijenga, On quartic surfaces in projective 3-space. 14th Dutch Mathematical Congress (Vrije Univ., Amsterdam, 1978). Nieuw Arch.Wisk. (3) 27 (1979), 98-103.

[18] A. Nobile, Families of curves on surfaces, Mathematische Zeitschrift, 187 (4) (1984), 453-470.

[19] G. Salmon, On the triple tangent planes to a surface of the third order, Cambridge and Dublin Math. J., 4 (1849), 252-260.

[20] L. Schläfli, On distribution of surfaces of the third order into species, Phil. Trans. Roy. Soc., 153 (1864), $193-247$.

[21] C. Segre, Étude des différentes surfaces du quatrième ordre à conique double ou cuspidale (générale ou décomposée) considèrées comme des projections de l'intersection de deux variétés quadratiques de l'espace à quatre dimensions, Math. Ann., 24 (1884), 313-444.

[22] D. Testa, The irreducibility of the spaces of rational curves on del Pezzo surfaces, J. Alg. Geom., 18 (1) (2009),. $37-61$.

[23] Y. Umezu, On normal projective surfaces with trivial dualizing sheaf, Tokyo J. Math., 4 (1981), (4), 343-354.

[24] Y. Umezu, Quartic surfaces of elliptic ruled type, Trans. Amer. Math. Soc., 283 (1984), (1), $127-1430$.

[25] T. Urabe, Classification of non-normal quartic surfaces, Tokyo J. Math., 9 (1986), (2), 265-295.

[26] P. Wagreich, Elliptic singularities of surfaces, Amer. J. Math. 92 (1970), 419-454.

[27] G. Xu, Subvarieties of general hypersurfaces in projective space, J. Differential Geom., 39(1) (1994), $139-172$.

Dipartimento di Matematica, Università degli Studi di Roma "Tor Vergata", Via della Ricerca Scientifica, 00133 ROMA, ITALY

E-mail address: cilibert@mat.uniroma2.it, flamini@mat.uniroma2.it

Université Grenoble I, Institut Fourier, UMR 5582 CNRS-UJF, BP 74, 38402 Saint Martin D'Hères CÉdex, FRANCE

E-mail address: mikhail.zaidenberg@ujf-grenoble.fr 